\title{
Open questions in the study of population III star formation
}

\author{
S. C. O. Glover ${ }^{1,2}$, P. C. Clark ${ }^{2}$, T. H. Greif ${ }^{2}$, J. L. Johnson ${ }^{3}$, \\ V. Bromm ${ }^{3}$, R. S. Klessen ${ }^{2}$ \& A. Stacy ${ }^{3}$ \\ ${ }^{1}$ Astrophysikalisches Institut Potsdam, An der Sternwarte 16, 14482 Potsdam, Germany \\ ${ }^{2}$ Institut für theoretische Astrophysik, Albert-Ueberle Strasse 2, 69120 Heidelberg, Germany \\ ${ }^{3}$ Department of Astronomy, University of Texas, Austin, TX 78712
}

\begin{abstract}
The first stars were key drivers of early cosmic evolution. We review the main physical elements of the current consensus view, positing that the first stars were predominantly very massive. We continue with a discussion of important open questions that confront the standard model. Among them are uncertainties in the atomic and molecular physics of the hydrogen and helium gas, the multiplicity of stars that form in minihalos, and the possible existence of two separate modes of metal-free star formation.
\end{abstract}

Keywords. astrochemistry, stars: formation, galaxies: formation, cosmology: theory

\section{Introduction}

We have learnt a great deal over the last decade concerning the formation of the first stars, the so-called population III or Pop III. A consensus has emerged regarding the properties of the protogalaxies (or 'minihalos') in which the first stars formed and the major physical and chemical processes involved in their formation. Their masses remain uncertain, but are widely expected to be significantly larger than the characteristic mass for present-day star formation. Good summaries of the present state of the field can be found in Bromm \& Larson (2004), Glover (2005) and Norman (2008). Nevertheless, there remain some important open questions. In this review, we discuss four of the most important of these issues and the efforts being made to resolve them. These issues are the impact of chemical rate coefficient uncertainties on the accuracy of our models of Pop III star formation; the perennial question of whether we have identified all of the important physical processes responsible for cooling the gas; the number of population III stars that form in each minihalo; and the question of whether population III actually consists of two sub-populations (Pop III.1 and Pop III.2) with different characteristic masses. Two further important issues - the question of what physical process terminates accretion onto the earliest protostars, and the impact of dark matter decay and annihilation on the formation of the first stars - are not discussed here as they are covered in detail elsewhere in these proceedings (see e.g. the contributions by Whalen, Tan, Freese \& Iocco).

\section{Population III star formation: the consensus view}

In the $\Lambda \mathrm{CDM}$ model for cosmic structure formation, the first gravitationally bound structures to form are very small (e.g. $M \sim M_{\odot}$ if cold dark matter consists of neutralinos; Diemand, Moore \& Stadel 2005) and consist purely of dark matter. Larger bound structures form through accretion and through the merger of these smaller objects, in a process known as hierarchical clustering. Once the mass of these gravitationally bound 
dark matter objects (conventionally referred to as dark matter halos) exceeds the cosmological Jeans mass, pressure forces can no longer prevent gas from falling into the potential wells created by the dark matter. Infalling gas is heated by adiabatic compression and by shocks, and in the absence of radiative cooling it will eventually reach a state of hydrostatic equilibrium, with a mean temperature given by the virial temperature of the halo, $T_{\text {vir }}$. The first dark matter halos to have masses greater than the cosmological Jeans mass have virial temperatures much smaller than the $\sim 10^{4} \mathrm{~K}$ temperature at which cooling from electronic excitation of atomic hydrogen becomes effective, and so the gas in these halos must rely on other, less effective forms of cooling.

It has long been recognized that the most important coolant at $T<10^{4} \mathrm{~K}$ in primordial gas is molecular hydrogen, $\mathrm{H}_{2}$ (Saslaw \& Zipoy 1967; Peebles \& Dicke 1968). In the absence of dust, this forms in the gas phase via the reaction chains

$$
\begin{aligned}
\mathrm{H}+\mathrm{e}^{-} & \rightarrow \mathrm{H}^{-}+\gamma, \\
\mathrm{H}^{-}+\mathrm{H} & \rightarrow \mathrm{H}_{2}+\mathrm{e}^{-},
\end{aligned}
$$

and

$$
\begin{aligned}
& \mathrm{H}+\mathrm{H}^{+} \rightarrow \mathrm{H}_{2}^{+}+\gamma \\
& \mathrm{H}_{2}^{+}+\mathrm{H} \rightarrow \mathrm{H}_{2}+\mathrm{H}^{+}
\end{aligned}
$$

with the $\mathrm{H}^{-}$mechanism generally dominating. Gas-phase $\mathrm{H}_{2}$ formation therefore relies on the presence of free electrons and protons, and the amount of $\mathrm{H}_{2}$ that can be formed by these reactions is limited by the recombination of the gas. A number of studies have examined the conditions required in order for the gas to form enough $\mathrm{H}_{2}$ to be able to cool within a Hubble time (see e.g. Haiman, Thoul, \& Loeb 1996; Tegmark et al. 1997; Yoshida et al. 2003), with the consensus being that virial temperatures $T_{\text {vir }} \gtrsim 1000 \mathrm{~K}$ are required, corresponding to halo masses of a few times $10^{5} \mathrm{M}_{\odot}$ or more.

The redshift at which the first halos of the required mass are formed depends to some extent of the definition of 'first'. The very first objects of this mass to form within a Hubble volume do so at redshifts $z \sim 50-60$ (Reed et al. 2005; Naoz, Noter \& Barkana 2006), but are exceedingly rare; on the other hand, the first object to form within a reasonable local volume, say 1 comoving $\mathrm{Mpc}^{3}$, does so at a somewhat lower redshift $z \sim 30$ (Glover 2005). For technical reasons, numerical simulations of population III star formation typically focus on the latter case.

If enough $\mathrm{H}_{2}$ can be formed to efficiently cool the gas, then it will undergo gravitational collapse. Initially, this collapse occurs rapidly. The gas temperature drops as the density increases, and so the gas becomes increasingly gravitationally unstable. However, as the collapse proceeds, cooling from $\mathrm{H}_{2}$ becomes steadily less efficient. The exponential fall-off in the $\mathrm{H}_{2}$ cooling rate at low temperatures prevents it from cooling the gas below $T \sim 200 \mathrm{~K}$, while the approach of the rotational and vibrational level populations of $\mathrm{H}_{2}$ to their local thermodynamic equilibrium (LTE) values at densities above $n_{\mathrm{cr}} \sim 10^{4} \mathrm{~cm}^{-3}$ renders $\mathrm{H}_{2}$ cooling inefficient at higher densities. The gas therefore accumulates in a quasi-hydrostatic "loitering state" with a characteristic temperature $T_{\text {char }}=200 \mathrm{~K}$ and characteristic density $n_{\text {char }}=10^{4} \mathrm{~cm}^{-3}$ (Abel, Bryan, \& Norman 2002; Bromm, Coppi, \& Larson 2002). Eventually, the amount of mass accumulated at this density exceeds the Bonnor-Ebert mass, which at this point is a few hundred $\mathrm{M}_{\odot}$, and the gravitational collapse resumes. However, beyond this point $\mathrm{H}_{2}$ cooling is unable to maintain the temperature at $T=200 \mathrm{~K}$; it steadily reheats, evolving with an effective polytropic index of $\gamma_{\text {eff }}=1.1$ (Omukai \& Nishi 1998). 
At densities $n>10^{8} \mathrm{~cm}^{-3}$, three-body formation of $\mathrm{H}_{2}$ via the reactions

$$
\begin{aligned}
\mathrm{H}+\mathrm{H}+\mathrm{H} & \rightarrow \mathrm{H}_{2}+\mathrm{H}, \\
\mathrm{H}+\mathrm{H}+\mathrm{H}_{2} & \rightarrow \mathrm{H}_{2}+\mathrm{H}_{2},
\end{aligned}
$$

becomes effective and rapidly converts all of the hydrogen to molecular form (Palla, Salpeter, \& Stahler 1983). Although the dramatic increase in the $\mathrm{H}_{2}$ abundance boosts the $\mathrm{H}_{2}$ cooling rate, the high gas density, the growing optical depth in the $\mathrm{H}_{2}$ rotational and vibrational lines (see e.g. Omukai \& Nishi 1998; Ripamonti et al. 2002; Ripamonti \& Abel 2004) and the heat input from $\mathrm{H}_{2}$ formation all combine to prevent the temperature from dropping significantly.

At densities $n \sim 10^{14} \mathrm{~cm}^{-3}$ and above, a second form of $\mathrm{H}_{2}$ cooling becomes important: $\mathrm{H}_{2}$ collision-induced emission (CIE). Although $\mathrm{H}_{2}$ has no permanent dipole moment, the complexes formed in collisions of $\mathrm{H}_{2}$ with $\mathrm{H}, \mathrm{H}_{2}$ or $\mathrm{He}$ can act as 'super-molecules', with non-zero dipole moments. Although these excited complexes last for only a very short time $\left(t_{\text {coll }} \sim 10^{-12} \mathrm{~s}\right.$; Ripamonti \& Abel 2004), there is nevertheless always a small probability that a photon will be emitted. At very high densities, collisions occur frequently enough to make this emission a viable means of cooling the gas. However, this CIE-dominated phase lasts for only a short time before the gas becomes optically thick in the continuum, and so CIE cooling is unable to significantly reduce the temperature.

At even higher densities $\left(n>10^{16} \mathrm{~cm}^{-3}\right)$, the gas becomes increasingly optically thick, and so radiative cooling becomes completely ineffective. However, collisional dissociation of $\mathrm{H}_{2}$ acts as a heat sink, keeping the temperature evolution of the gas close to isothermal until most of the $\mathrm{H}_{2}$ has been destroyed (Omukai et al. 2005; Turk, Abel \& O'Shea 2008). This occurs by the time that the density reaches $10^{21} \mathrm{~cm}^{-3}$, and beyond this point the evolution of the gas becomes adiabatic. This is the moment at which we first have something that we can identify as a true protostar.

At the moment that this protostar forms, its mass is less than $0.01 \mathrm{M}_{\odot}$, but it is surrounded by a dense, massive envelope of infalling gas with a mass of hundreds of solar masses. Accretion of this envelope is expected to occur at a rapid rate: a simple scaling argument suggests that the accretion rate should scale as $\dot{M} \propto c_{\mathrm{s}}^{3} \propto T^{3 / 2}$, and since the temperature of the gas is between 10-100 times larger than in local star forming regions, the expected accretion rates are orders of magnitude larger than the rate of order $10^{-5} \mathrm{M}_{\odot} \mathrm{yr}^{-1}$ that is typical locally. Accretion rates have been estimated using a variety of techniques (see Glover 2005, section 4), and although the estimated rates differ somewhat, in every case one expects the star to be able to accrete more than $100 \mathrm{M}_{\odot}$ of gas within the Kelvin-Helmholtz relaxation time. Moreover, stellar feedback in the form of radiation-driven winds is expected to be far less effective in primordial gas than in metal-enriched gas (see e.g. Kudritzki 2002), and so there seems little to prevent the star from becoming massive.

Hence, population III stars are expected to be massive and short-lived, surviving for only a few million years. How they end their lives depends on both their mass and the speed at which they are rotating. Non-rotating population III stars with masses in the range $10-50 \mathrm{M}_{\odot}$ are expected to explode as either conventional type II supernovae or as hypernovae (Umeda \& Nomoto 2002). For masses between 50 and $140 \mathrm{M}_{\odot}$ and above $260 \mathrm{M}_{\odot}$, direct collapse to form a black hole is expected, while non-rotating Pop III stars with masses in the range $140-260 \mathrm{M}_{\odot}$ are expected to end their lives as pair-instability supernovae (Heger \& Woosley 2002). Rapid rotation changes this picture somewhat, by enhancing the effects of mass-loss, and by inducing mixing within the star. The latter effect leads to the star having a larger helium core at the end of its main sequence 
evolution, and so likely allows less massive stars to become pair instability supernovae (Ekström et al. 2008).

\section{Open questions}

\subsection{Are uncertainties in the chemical rate coefficients important?}

Our ability to construct accurate models of the chemical evolution of metal-free gas is constrained by the level of accuracy with which the rate coefficients of the key chemical reactions have been determined. This varies significantly depending on the reaction in question. Many of the most important reactions involved in the formation and destruction of $\mathrm{H}_{2}$ have rate coefficients that have been determined to within an accuracy of the order of 10-20 \% at typical protogalactic temperatures (Abel et al. 1997; Galli \& Palla 1998). However, there are several important reactions whose rates are far more uncertain.

One example is the charge transfer reaction

$$
\mathrm{H}_{2}+\mathrm{H}^{+} \rightarrow \mathrm{H}_{2}^{+}+\mathrm{H}
$$

which is a major destruction pathway for $\mathrm{H}_{2}$ in hot, ionized gas. Savin et al. (2004) present a new calculation of the rate of this reaction, and show that previous determinations, some of which remain widely used in the literature, differ by orders of magnitude. Fortunately, this process is unimportant in cold gas, owing to its large endothermicity, and so the large uncertainty in this reaction has little impact on the accuracy with which we can model the formation of the first stars. However, its impact on the formation of so-called Pop III.2 stars (discussed in more detail in $§ 3.4$ below) may be much larger and deserves further study.

Another source of uncertainty stems from the competition between two of the main destruction pathways for $\mathrm{H}^{-}$, associative detachment (reaction 2.2 above) and mutual neutralization

$$
\mathrm{H}^{-}+\mathrm{H}^{+} \rightarrow \mathrm{H}+\mathrm{H}
$$

Glover, Savin \& Jappsen (2006) surveyed the literature available on the rates of these reactions at low temperatures $\left(T<10^{4} \mathrm{~K}\right)$, and showed that both were uncertain by an order of magnitude. They also examined the effects of this uncertainty on the chemistry, cooling and dynamics of the gas. In the conventional Pop III formation scenario, the fractional ionization of the gas is small enough to ensure that associative detachment always dominates over mutual neutralization, and so any uncertainty in the rate coefficients has almost no effect. In gas cooling from a highly ionized state, however, as in some of the Pop III.2 scenarios discussed in section 3.4 below, mutual neutralization dominates initially, with associative detachment becoming important only once the gas has recombined sufficiently. In this case, any uncertainty in the rates of these two reactions leads to an uncertainty in the $\mathrm{H}_{2}$ formation rate, and in the final amount of $\mathrm{H}_{2}$ formed. More recently, Glover \& Abel (2008) have shown that this uncertainty affects the amount of HD that can form, and so also influences the minimum temperature that the collapsing gas can reach.

Fortunately, experimentalists have begun to address this source of uncertainty. Recent measurements of the mutual neutralization reaction rate at low temperatures by Xavier Urbain have reduced what was an order of magnitude uncertainty to something closer to a $50 \%$ uncertainty (X. Urbain, private communication). At the same time, an experiment designed to accurately measure the rate coefficient for the associative detachment reaction has been funded and is currently under construction (Bruhns et al. 2008; D. Savin, private 
communication), and so this source of uncertainty may also have been removed in a few years time.

Finally, and perhaps most importantly, a very large uncertainty exists in the rate of the three-body $\mathrm{H}_{2}$ formation reaction. The current state of the literature regarding the rate of reaction 2.5 was surveyed in Glover (2008), who showed that in the temperature range $200<T<2000 \mathrm{~K}$ relevant for population III star formation, there is an uncertainty in the rate coefficient of two to three orders of magnitude. Moreover, there is no sign that this uncertainty is reducing: indeed, the two most recent determinations of the rate coefficient (by Abel, Bryan, \& Norman 2002 and by Flower \& Harris 2007) are the two with the greatest disagreement. The uncertainty in the rate of reaction 2.6 is harder to quantify, as there have been fewer studies made of this reaction. However, if we assume (following Jacobs, Giedt \& Cohen 1967) that the rate of this reaction is $1 / 8$ th that of reaction 2.5, then the obvious implication is that this rate has a similarly large uncertainty.

The effects of the uncertainty in the three-body $\mathrm{H}_{2}$ formation rate on the thermal evolution of the gas have been examined by Glover \& Abel (2008) and Glover \& Savin (2008) using highly simplified one-zone models. These studies find that the rate coefficient uncertainties lead to an uncertainty of approximately $50 \%$ in the temperature evolution of the gas in the density range $10^{8}<n<10^{13} \mathrm{~cm}^{-3}$. The effect of this uncertainty on the dynamical evolution of the gas and in particular on the predicted protostellar accretion rates are not currently known, although work is currently under way to address this.

\subsection{Are we including all of the significant coolants?}

As previously noted, $\mathrm{H}_{2}$ has long been recognized as the most important coolant in primordial gas at temperatures $T<10^{4} \mathrm{~K}$. At the same time, it is clear from our previous discussion that $\mathrm{H}_{2}$ becomes increasingly ineffective as a coolant as we move to higher densities, owing to the low critical density at which its rotational and vibrational level populations reach their LTE values, and to the fact that at densities $n \gtrsim 10^{10} \mathrm{~cm}^{-3}$, optical depth effects further suppress $\mathrm{H}_{2}$ cooling.

The comparative ineffectiveness of $\mathrm{H}_{2}$ as a coolant in high density metal-free gas has motivated various authors to examine the role that might be played by other coolants at high densities. Perhaps the best studied alternative coolant is hydrogen deuteride, HD. It has excited rotational and vibrational levels that have radiative lifetimes that are about a factor of 100 shorter than those of $\mathrm{H}_{2}$, and so the $\mathrm{HD}$ cooling rate does not reach its LTE limit until $n \sim 10^{6} \mathrm{~cm}^{-3}$. It is also a far more effective coolant than $\mathrm{H}_{2}$ at low temperatures ( $T \lesssim 200 \mathrm{~K}$; see e.g., Flower et al. 2000). This is due primarily to the fact that radiative transitions can occur between rotational levels with odd and even values of $J$, allowing cooling to occur through the $J=1 \rightarrow 0$ transition. The corresponding odd $\leftrightarrow$ even transitions in the case of $\mathrm{H}_{2}$ represent conversions from ortho- $\mathrm{H}_{2}$ to para- $\mathrm{H}_{2}$ or vice versa, and are highly forbidden. Furthermore, at low temperatures the ratio of $\mathrm{HD}$ to $\mathrm{H}_{2}$ can be significantly enhanced with respect to the cosmological D:H ratio by chemical fractionation (see e.g., Glover 2008).

The role of HD cooling in early minihalos has been investigated by a number of authors. In the case of the earliest generation of minihalos, which form from very cold neutral gas that is never heated to more than a few thousand $\mathrm{K}$ during the course of the galaxy formation process, the importance of HD appears to be a function of the size and dynamical history of the minihalo (Ripamonti 2007; McGreer \& Bryan 2008). In small minihalos $\left(M \lesssim 10^{6} \mathrm{M}_{\odot}\right)$ that collapse in an unperturbed, relatively uniform fashion, $\mathrm{H}_{2}$ cooling can lower the temperature of the gas enough to allow HD (which is strongly enhanced at low temperatures by chemical fractionation; Glover 2008) to take over and dominate the cooling. In larger minihalos $\left(M \gtrsim 10^{6} \mathrm{M}_{\odot}\right)$ that have a more complex dynamical history, 
$\mathrm{H}_{2}$ cooling is unable to lower the temperature to the same extent, and so the gas never becomes cold enough for HD to dominate. In this case, it contributes no more than about 20-30\% of the total cooling (Glover \& Savin 2008) and does not appear to significantly affect the dynamics of the gas (Bromm, Coppi, \& Larson 2002). HD cooling is also of great importance in situations where an increase in the fractional ionization of the gas has led to an increase in the $\mathrm{H}_{2}$ fraction. In this situation, the gas often becomes cool enough for HD to dominate (see e.g., Nakamura \& Umemura 2002; Nagakura \& Omukai 2005; Johnson \& Bromm 2006; Shchekinov \& Vasiliev 2006). This scenario is discussed in more detail in $\S 3.4$ below.

Another molecule to have attracted considerable attention is lithium hydride, $\mathrm{LiH}$. This molecule has a very large dipole moment, $\mu=5.888$ debyes (Zemke \& Stwalley 1980), and consequently its excited levels have very short radiative lifetimes. Therefore, despite the very low lithium abundance in primordial gas $\left(x_{\mathrm{Li}}=4.3 \times 10^{-10}\right.$, by number; see Cyburt 2004), it was thought for a time that $\mathrm{LiH}$ would dominate the cooling at very high densities (see e.g., Lepp \& Shull 1984). However, accurate quantal calculations of the rate of formation of $\mathrm{LiH}$ by radiative association (Dalgarno et al. 1996; Gianturco \& Gori Giorgi 1996; Bennett et al. 2003)

$$
\mathrm{Li}+\mathrm{H} \rightarrow \mathrm{LiH}+\gamma,
$$

have shown that the rate is much smaller than was initially assumed, while recent work by Defazio et al. (2005) has shown that the reaction

$$
\mathrm{LiH}+\mathrm{H} \rightarrow \mathrm{Li}+\mathrm{H}_{2},
$$

has no activation energy and so will be an efficient destruction mechanism for LiH for as long as some atomic hydrogen remains in the gas. Consequently, the amount of lithium hydride present in the gas is predicted to be very small, even at very high densities, and so LiH cooling is no longer believed to be important (Mizusawa, Omukai, \& Nishi 2005; Glover \& Savin 2008).

Finally, molecular ions such as $\mathrm{H}_{2}^{+}, \mathrm{H}_{3}^{+}$or $\mathrm{HeH}^{+}$provide another possible source of cooling in dense primordial gas. Early work on $\mathrm{H}_{2}^{+}$cooling in ionized primordial gas can be found in Suchkov \& Shchekinov $(1977,1978)$, and its possible importance in hot, highly ionized conditions has recently been re-emphasized by Yoshida et al. (2007). However, it has a low critical density $\left(n_{\mathrm{cr}} \sim 10^{3} \mathrm{~cm}^{-3}\right)$ and is also readily destroyed in collisions with atomic hydrogen

$$
\mathrm{H}_{2}^{+}+\mathrm{H} \rightarrow \mathrm{H}_{2}+\mathrm{H}^{+} .
$$

These factors make it unlikely to be an important coolant at high densities.

$\mathrm{HeH}^{+}$is a more promising candidate: it has a large dipole moment, a large cooling rate per molecule when in LTE, and hence a very large critical density (Engel et al. 2005). However, once again it is readily destroyed in collisions with atomic hydrogen

$$
\mathrm{HeH}^{+}+\mathrm{H} \rightarrow \mathrm{H}_{2}^{+}+\mathrm{He}
$$

and so its abundance in high density gas is very small (Glover \& Savin 2008). It therefore never becomes a significant coolant.

The last of these three molecular ions, $\mathrm{H}_{3}^{+}$, is perhaps the most interesting. It has a large cooling rate per molecule when in LTE (Neale, Miller, \& Tennyson 1996) and hence a large critical density (Glover \& Savin 2006, 2008). Unlike $\mathrm{H}_{2}^{+}$and $\mathrm{HeH}^{+}$, it is not readily destroyed by collisions with atomic hydrogen - the reaction

$$
\mathrm{H}_{3}^{+}+\mathrm{H} \rightarrow \mathrm{H}_{2}^{+}+\mathrm{H}_{2}
$$


does occur, but must overcome a large energy barrier, and so proceeds slowly at temperatures $T<1000 \mathrm{~K}$. Moreover, $\mathrm{H}_{3}^{+}$is known to be an important coolant in at least one astrophysical scenario, namely in the upper atmospheres of gas giants (Miller et al. 2000). Glover \& Savin (2008) have examined in detail the role that $\mathrm{H}_{3}^{+}$plays in the cooling of primordial gas. They find that in most variations of the conventional Pop III.1 formation scenario, $\mathrm{H}_{3}^{+}$comes close to being an important coolant, but never quite succeeds. It contributes to the total cooling rate at densities $10^{7}<n<10^{9} \mathrm{~cm}^{-3}$ at the level of a few percent, making it the third most important coolant after $\mathrm{H}_{2}$ and $\mathrm{HD}$, but unimportant for the overall thermal evolution of the gas. However, Glover \& Savin (2008) do identify one scenario in which $\mathrm{H}_{3}^{+}$can become the dominant coolant. If a significant ionization rate can be maintained at densities $n>10^{8} \mathrm{~cm}^{-3}$, for instance by cosmic rays or very hard X-rays, then ionization of $\mathrm{H}_{2}$ to $\mathrm{H}_{2}^{+}$is quickly followed by the reaction

$$
\mathrm{H}_{2}^{+}+\mathrm{H}_{2} \rightarrow \mathrm{H}_{3}^{+}+\mathrm{H}
$$

resulting in the production of a large number of $\mathrm{H}_{3}^{+}$ions. In this scenario, the $\mathrm{H}_{3}^{+}$abundance can be maintained at a high enough level to allow $\mathrm{H}_{3}^{+}$to dominate the cooling rate. The required ionization rate is of the order of $10^{-17} \mathrm{~s}^{-1}$. This is far larger than could be produced by plausible extragalactic sources, but is perhaps consistent with production by local sources. An interesting possibility in this context is that dark matter annihilation within the dense core may provide the necessary source of ionization.

\subsection{How many stars form per minihalo?}

High resolution AMR and SPH simulations of the formation of the first stars typically find that only a single collapsing protostellar core forms in each minihalo (see e.g. Abel, Bryan, \& Norman 2002; Yoshida et al. 2006; O'Shea \& Norman 2007). However, it is possible that this result is a consequence of the numerical methods used to simulate the gas, rather than of the gas physics. In order to properly resolve the gravitational collapse of the gas, it is necessary to ensure that the gravitational Jeans length is resolved with sufficient computational elements, a criterion that has been formalized by Truelove et al. (1997) for grid-based codes and by Bate \& Burkert (1997) for SPH. If the gas remains close to isothermal during the collapse, then the Jeans length will continually decrease, as will the Courant timestep, the largest timestep on which the hydrodynamical evolution can be followed while still maintaining numerical stability. Therefore, once the gas reaches very high densities, the simulations can take only very small timesteps, and it is common practice in the numerical study of population III star formation to terminate the simulations at this point. However, this practice means that the simulations can follow the evolution of multiple collapsing objects only if the collapses are very closely synchronized in time. In reality, we know from the numerical study of local star formation that gravitational fragmentation is rarely so well synchronized. Typically, there is always some region with a higher density, or a lower angular momentum, that collapses first, with other objects forming only after a few local dynamical times. For example, the overall duration of star formation in nearby molecular clouds is found to be comparable to the global crossing time of the cloud (e.g. Elmegreen 2000; Mac Low \& Klessen 2004) and exceeds the collapse timescale of individual stars by one to two orders of magnitude. If only the initial collapse is simulated, the formation of these other objects can be missed. To avoid this problem, it is common in numerical studies of local star formation to replace gas which has collapsed beyond the limiting resolution of the simulation with artifical sink particles. These particles possess the mass and linear momentum of the gas that they replace, and continue to interact with the surrounding gas via gravity. They are able to accrete additional infalling gas, provided that it is gravitationally bound 
to them and comes within a preset accretion radius. However, they no longer feel the effects of hydrodynamical pressure gradients, and the subsequent evolution of the gas incorporated into them is not followed (Bate, Bonnell, \& Price 1995). By replacing high density, unresolved gas with sink particles, it becomes possible to follow the process of gravitational fragmentation for many dynamical times.

Despite their wide usage in the study of present-day star formation, sink particles have been used in only a few studies of primordial star formation. Bromm, Coppi, \& Larson (1999, 2002) used sink particles in their study of Pop III star formation, creating them once the gas density exceeded $n_{\mathrm{th}}=10^{8} \mathrm{~cm}^{-3}$. They found that several massive clumps formed in most of their simulations. The only case for which this was not true was for the smallest mass halo they simulated $\left(M_{\text {tot }}=2 \times 10^{5} \mathrm{M}_{\odot}\right)$, in which pressure forces would be expected to have the greatest effect. Taken at face value, these results suggest that the formation of several massive stars per minihalo could be a common outcome of population III star formation. However, the initial conditions used in these simulations - specifically, the adoption of a solid-body initial rotation profile - have been criticized on the grounds that they are overly prone to fragmentation (Jappsen et al. 2007). The large, rotationallysupported disks formed in the Bromm, Coppi, \& Larson $(1999,2002)$ simulations are not seen in simulations that start from self-consistent cosmological initial conditions (e.g. Abel, Bryan, \& Norman 2002; Yoshida et al. 2006), and so the fragmentation may also not occur.

A second study to utilize sink particles was that of Bromm \& Loeb (2004). They adopted similar initial conditions to Bromm, Coppi, \& Larson (2002), but used a numerical technique called particle splitting (Kitsionas \& Whitworth 2002; Bromm \& Loeb 2003) to allow them to follow the evolution of the first dense clump up to a much higher density $\left(n_{\mathrm{th}}=10^{12} \mathrm{~cm}^{-3}\right)$. They found no evidence for sub-fragmentation of this dense clump on timescales $t \lesssim 10^{4} \mathrm{yr}$ after the formation of the central sink particle.

More recently, Clark, Glover \& Klessen (2008) used SPH with sink particles to simulate the collapse of dense protostellar cores of various metallicities. Their simulations followed collapse from an initial density of $5 \times 10^{5} \mathrm{~cm}^{-3}$ up to a density of $n_{\mathrm{th}} \sim 10^{17} \mathrm{~cm}^{-3}$. The highest resolution simulation used 25 million $\mathrm{SPH}$ particles to represent $500 \mathrm{M}_{\odot}$, and so had a mass resolution of $M_{\text {res }}=2 \times 10^{-3} \mathrm{M}_{\odot}$. The thermodynamic evolution of the gas was treated using a tabulated equation of state, based on the one-zone results of Omukai et al. (2005). The initial rotational and turbulent energies were chosen to be consistent with the results of previous studies of Pop III star formation, such as Abel, Bryan, \& Norman (2002). Although the primary focus of Clark et al.'s study was an examination of the effects of metal enrichment, they also modelled a $Z=0$ core for comparison. They found that even in the primordial case, the core fragmented, forming of the order of 20 sink particles. The mass function of these fragments was considerably flatter than the present day IMF, implying that most of the mass was concentrated in the few most massive fragments. Clark et al. also found that there was a delay of several local freefall times between the formation of the first and second sink particles, and that at the time that the first sink particle formed, the radial profiles of mass density and specific angular momentum were similar to those seen in previous high-resolution simulations performed without sink particles. Slices through the densest structure at the time that the first sink particle forms also show little sign of the impending fragmentation (see e.g. Figure 1). These results support the view that simulations without sink particles (or some comparable treatment of unresolved gas) run the risk of missing the formation of all but the first protostar.

Careful analysis of the Omukai et al. (2005) equation of state for zero-metallicity gas shows roughly isothermal behavior in the density range $10^{14} \mathrm{~cm}^{-3} \leqslant n \leqslant 10^{16} \mathrm{~cm}^{-3}$, i.e. 


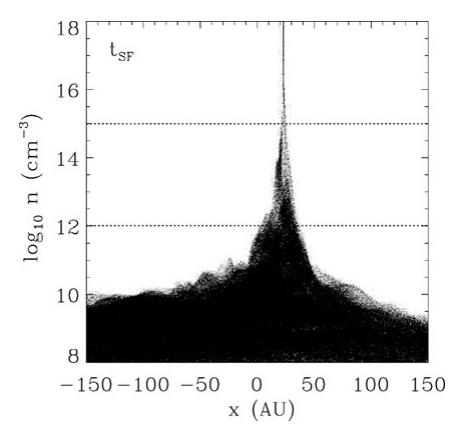

Figure 1. The densities of the SPH particles in the Clark, Glover \& Klessen (2008) high resolution simulation, plotted as a function of their $\mathrm{x}$-position at the moment that the first sink particle forms. Only the particles contained within the central $300 \mathrm{AU}$ of the simulation are shown. At this point, there is little sign of the secondary fragmentation that will shortly occur.

just before the gas becomes optically thick and begins to heat up adiabatically. Conservation of angular momentum during this phase of the collapses leads to the build-up of a rotationally supported massive disk-like structure, which becomes gravitationally unstable and fragments. This is understandable, as isothermal disks are susceptible to gravitational instability (Bodenheimer 1995) once they have accumulated sufficient mass. Further, Goodwin, Whitworth \& Ward-Thompson (2004a,b) show how even very low levels of turbulence can induce fragmentation. Since turbulence creates local anisotropies in the angular momentum on all scales, it can always provide some centrifugal support against gravitational collapse. This support can then provide a window in which fragmentation can occur. Moreover, the density at which this occurs is significantly higher than the maximum density resolved in the earlier Bromm \& Loeb (2004) simulation, and we would therefore not expect the fragmentation to have been observed in that simulation.

However, the results of the Clark, Glover \& Klessen (2008) simulations come with several caveats attached. The most important relates to the use of a tabulated equation of state to represent the thermodynamic evolution. In this approach, one is essentially assuming that as the density of the gas changes, the gas temperature changes instantaneously to reflect the behaviour prescribed by the equation of state. In reality, the gas temperature will adjust itself on a timescale $t_{\text {cool }}$, which in primordial gas is comparable to or greater than the dynamical timescale. This delay may damp out density fluctuations, thereby helping to suppress fragmentation. A second concern is that the Clark, Glover \& Klessen (2008) simulations do not allow sink particles to merge, and so may overestimate the number of fragments that survive in reality. However, there are two reasons for believing that this is not a major concern. Firstly, the sink particle volume is considerably larger than the actual protostellar object in its center and so the cross section for two real protostars to collide is orders of magnitude smaller than the geometric cross section of the sink particles. Secondly, Clarke \& Bonnell (2008) have demonstrated that the importance of collisions depends on the balance between shinkage of the cluster core by adiabiatic contraction and puffing via collisional relaxation. As a result of this balance, collisions will start to affect the mass function in the Clark, Glover \& Klessen (2008) cluster only after $10^{3}$ to $10^{4}$ objects have been formed, further along in its evolution than has yet been simulated.

A final concern is that the Clark et al. simulations do not account for the effects of feedback from the protostars that have already formed. Since fragmentation seems to occur on a timescale much shorter than the protostellar Kelvin-Helmholtz timescale, 
feedback from the protostar is probably unimportant. However, this assumption needs to be verified.

To summarize, there is some evidence, primarily from the work by Bromm, Coppi, \& Larson (2002) and Clark, Glover \& Klessen (2008), that the number of population III stars that form in the earliest minihalos is higher than the single star that is commonly assumed. However, this conclusion remains controversial, and the evidence is not yet convincing.

\subsection{Is there a population III.2?}

The final issue that we discuss in this review is the question of whether there might be more than one mode of population III star formation. As we have already discussed, the first Pop III stars form in minihaloes in which $\mathrm{H}_{2}$ dominates the cooling, with HD generally playing only a minor role. However, work by a number of authors has shown that if molecular hydrogen formation can be more efficiently catalyzed by a higher electron abundance, then HD cooling of the gas can become efficient, and can allow the gas temperature to reach values close to the floor set by the CMB (see e.g. Nakamura \& Umemura 2002; Nagakura \& Omukai 2005; Johnson \& Bromm 2006; Yoshida et al. 2007). Efficient HD cooling lowers the characteristic gravitational fragmentation mass scale, and may also reduce the accretion rate of gas onto the fragment or fragments that form (Yoshida et al. 2007; McGreer \& Bryan 2008). It is therefore reasonable to assume that if $\mathrm{HD}$ cooling becomes dominant, lower mass stars will be formed than in the case in which $\mathrm{H}_{2}$ dominates throughout. If this is true, then it suggests that there may be two distinct sub-populations of stars within population III: a first generation of stars forming from undisturbed primordial gas (termed population III.1 by Tan \& McKee 2008) and a subsequent generation forming from gas that remains metal-free but that has an elevated fractional ionization, owing to either the infall of the gas into the deeper potential wells of the first galaxies, or to the effects of feedback from the first generation of stars. Tan $\&$ McKee (2008) term this second generation 'population III.2'.

There are various different scenarios that may lead to the formation of population III.2 stars. The simplest scenario involves primordial star formation in the first galaxies (Greif $\&$ Bromm 2006). If the virial temperature of such an object exceeds $10^{4} \mathrm{~K}$, then most infalling gas will be shock-heated to temperatures high enough for it to become ionized. As this gas subsequently cools and recombines, it will form $\mathrm{H}_{2}$ at an accelerated rate, owing to the enhanced fractional ionization of the gas (Shapiro \& Kang 1987). If enough $\mathrm{H}_{2}$ is formed to cool the gas to roughly $150 \mathrm{~K}$, then HD cooling will take over, driving the temperature down towards the CMB floor. Recent simulations of the formation of the first galaxies by Greif et al. (2008) show this mechanism in operation (see Fig. 2). However, this scenario can only produce population III.2 stars if the gas forming these galaxies has remained metal-free. Greif et al. (2008) demonstrate that these galaxies typically have of order 10 or more progenitors that have undergone population III.1 star formation, and so it is likely that most of their gas will already have been contaminated with metals. Nevertheless, population III.2 stars may still be able to form in these objects if metal mixing is inefficient, or if some fraction of the gas that they accrete has remained pristine.

The Pop III.2 star formation mode may also be triggered by the explosions of the first supernovae (SN), as the shocks from these explosions can heat and ionize primordial gas (e.g. Mackey, Bromm \& Hernquist 2003; Salvaterra, Ferrara \& Schneider 2004; Machida et al. 2005; Johnson \& Bromm 2006). In a three-dimensional simulation of the explosion of a Pop III pair-instability SN, Greif et al. (2007) found that the SN shock-compression of the gas in minihalos can speed its collapse. However, in this simulation the neighboring 

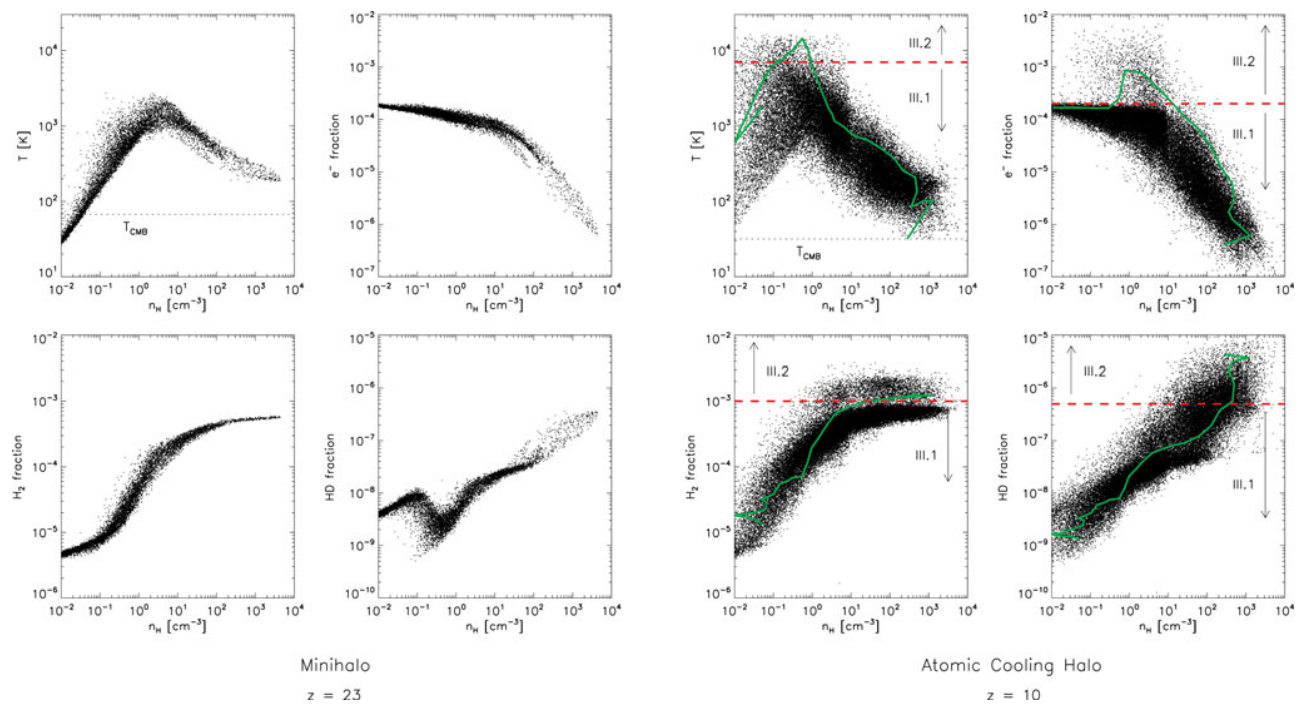

Figure 2. The phase-space distribution of gas inside a minihalo (left-hand panel) and an atomic cooling halo (right-hand panel). We show the temperature, electron fraction, HD fraction and $\mathrm{H}_{2}$ fraction as a function of hydrogen number density, clockwise from top left to bottom left. Left-hand panel: In the minihalo case, adiabatic collapse drives the temperature to $>10^{3} \mathrm{~K}$ and the density to $n_{\mathrm{H}}>1 \mathrm{~cm}^{-3}$, where molecule formation sets in and allows the gas to cool to $\simeq 200 \mathrm{~K}$. At this point, the central clump becomes Jeans-unstable and ultimately forms a Pop III.1 star. Right-hand panel: In the galaxy, a second cooling channel has emerged due to an elevated electron fraction at the virial shock, which in turn enhances molecule formation and allows the gas to cool to the temperature of the CMB. The dashed red lines and arrows approximately delineate the resulting Pop III.1 and Pop III.2 channels, while the solid green lines denote the path of a representative fluid element that follows the Pop III.2 channel.

minihalo was not strongly shocked and so did not yield any evidence for the formation of Pop III.2 stars, although it is noted that minihalos within perhaps $<500 \mathrm{pc}$ of such a SN could become strongly ionized. Such minihalos would be the likely sites of the formation of Pop III.2 stars which are formed in the wake of the first SN. Simulations of SN feedback on close-by minihalos which account for the mixing of the metals injected by the supernova with the primordial gas are an important next step (see Cen \& Riquelme 2007).

A further location in which population III.2 stars may be formed is in the relic H II regions left behind after the death of the first massive Pop. III stars. Again, the gas starts in a hot and highly ionized state, and forms significant quantities of $\mathrm{H}_{2}$ as it cools and recombines (Ricotti, Gnedin \& Shull 2002; Johnson, Greif, \& Bromm 2007; Yoshida, Omukai \& Hernquist 2007). However, the H II regions produced by the first massive stars are generally capable of expelling most of the gas from the galaxies containing the stars, and expanding to fill large volumes in the low-density intergalactic medium. Therefore, most of the $\mathrm{H}_{2}$ that forms within the relic $\mathrm{H}$ II regions resides at very low densities and is initially unavailable for star formation.

Finally, Pop. III.2 stars may also be formed in primordial gas irradiated with a sufficiently strong flux of cosmic rays. Stacy \& Bromm (2007) studied the effects of cosmic rays on population III star formation and showed that the enhanced fractional ionization that they create would lead to enhanced $\mathrm{H}_{2}$ and $\mathrm{HD}$ production and the cooling of the gas to $T \sim T_{\mathrm{CMB}}$ for cosmic ray ionization rates greater than $\zeta \sim 10^{-19} \mathrm{~s}^{-1}$. Jasche, Ciardi \& Ensslin (2007) reached similar conclusions in a separate study. 
As all three scenarios rely on the gas forming more $\mathrm{H}_{2}$ than in the standard Pop. III.1 formation scenario, additional physical effects that reduce the amount of $\mathrm{H}_{2}$ formed will lessen the likelihood that any Pop. III.2 stars form. One such effect is radiative cooling by $\mathrm{H}_{2}-\mathrm{H}^{+}$and $\mathrm{H}_{2}-\mathrm{e}^{-}$collisions. Glover \& Abel (2008) include these processes in their models of primordial gas cooling and show that, somewhat counter-intuitively, the increased cooling that they provide at early times (while the fractional ionization is high) leads to less $\mathrm{H}_{2}$ being formed, and hence to less cooling at late times. This surprising result is a consequence of the different temperature dependences of the rate coefficients for $\mathrm{H}^{-}$formation and for $\mathrm{H}^{+}$recombination. Decreasing the temperature decreases the rate at which $\mathrm{H}^{-}$forms, and hence decreases the $\mathrm{H}_{2}$ formation rate. However, it also increases the $\mathrm{H}^{+}$recombination rate, and so reduces the time available for $\mathrm{H}_{2}$ formation before the necessary electrons are lost from the gas. Therefore, the faster the gas cools at early times, while the fractional ionization remains large, the less $\mathrm{H}_{2}$ it will ultimately form. Glover \& Abel (2008) show that in their one-zone calculations, this effect does not prevent the gas from cooling below $100 \mathrm{~K}$; however, its effects in more realistic situations have not yet been investigated.

Another obvious candidate for suppressing Pop III.2 star formation is the far ultraviolet background built up by the first stars (Haiman, Abel \& Rees 2000; Machacek, Bryan \& Abel 2001; Ricotti, Gnedin \& Shull 2002; Ahn et al. 2008). This dissociates $\mathrm{H}_{2}$ and HD, and so acts to suppress cooling. Yoshida, Omukai \& Hernquist (2007) estimate that a far-UV flux of only $3 \times 10^{-22} \mathrm{erg} \mathrm{s}^{-1} \mathrm{~cm}^{-2} \mathrm{~Hz}^{-1} \mathrm{sr}^{-1}$ is required in order to prevent the gas from cooling below $200 \mathrm{~K}$, thereby completely suppressing the formation of Pop. III.2 stars. However, this estimate is based on simple one-zone calculations that are unlikely to properly capture the dynamics of the gas, and so may be misleading. Furthermore, in galaxies in which the virial temperature exceeds $\sim 10^{4} \mathrm{~K}$ the column density of $\mathrm{H}_{2}$ may become high enough to shield the central regions from the far ultraviolet background radiation, thereby allowing $\mathrm{HD}$ molecules to survive and to be an important coolant (see Johnson et al. 2008). An investigation of the effects of the ultraviolet background on HD cooling and the formation of Pop. III.2 using a fully three-dimensional approach would be very valuable.

\section{Outlook}

Of the four open questions discussed in this review, those involving uncertainties in the chemistry and in the cooling of metal-free gas seem the easiest to address. Existing work has gone a long way towards establishing the effects of the chemical uncertainties, and scientists from the atomic and molecular physics communities are rising to the challenge that these uncertainties present. As far as the cooling is concerned, we now have a basic understanding of which processes are important and which are unimportant over a very wide range of scales during protostellar collapse. While there may still be occasional surprises, such as the importance of $\mathrm{H}_{2}-\mathrm{H}^{+}$and $\mathrm{H}_{2}-\mathrm{e}^{-}$collisions in gas with only a slightly elevated fractional ionization, we think it unlikely that any of these will fundamentally change our picture of population III star formation.

The question of how many population III stars form in each minihalo is much further from being settled. There is now reason to believe that the conventional wisdom that only one massive star forms per minihalo may be incorrect. On the other hand, it may be that it is the simulations that are incorrect; they may be giving us a misleading view of what happens owing to the approximations that they make. To settle this question, further numerical study is required, using methods that are capable of following the hydrodynamical evolution of the gas beyond the point at which the first protostar forms, 
but that do not make as many approximations as in the Clark, Glover \& Klessen (2008) study.

Finally, the question of whether Pop. III.2 exists as a distinct sub-population within population III also presents continuing difficulties. Some uncertainties, such as the impact of the revised treatment of $\mathrm{H}_{2}$ cooling presented by Glover \& Abel (2008), will be easy to address with the next generation of numerical simulations, and so should be resolved within the next year or so. However, other issues, such as the impact of the extragalactic far ultraviolet background, involve physics that is difficult to simulate accurately, and it will take far longer before we fully understand its effects. (As an example, consider that after more than ten years of study, there is still not complete agreement regarding the degree to which the ultraviolet background regulates $\mathrm{H}_{2}$ cooling in minihalos; c.f. Haiman, Rees \& Loeb 1997; Haiman, Abel \& Rees 2000; Ricotti, Gnedin \& Shull 2002; Wise \& Abel 2007; O'Shea \& Norman 2008). Furthermore, even after these issues are addressed, we will still not be able to claim with confidence that Pop. III.1 and Pop. III.2 differ until we have a better understanding of the processes regulating accretion onto population III stars. Ultimately, this may be a question that is answered as much by stellar archaeology as by theoretical study.

\section{Acknowledgements}

The authors would like to thank the organisers of IAU Symposium 255 for organising a very stimulating and enjoyable meeting. RSK acknowledges partial support from the Emmy Noether grant KL 1358/1. RSK, TG, and PCC also acknowledge support from the DFG SFB 439 'Galaxies in the Early Universe'. TG would also like to thank the Heidelberg Graduate School of Fundamental Physics (HGSFP) for financial support. The HGSFP is funded by the excellence initiative of the German government (grant number GSC 129/1). VB acknowledges support from NSF grant AST-0708795.

\section{References}

Abel, T., Anninos, P., Zhang, Y., \& Norman, M. L. 1997, New Astron., 2, 181

Abel, T., Bryan, G. L., \& Norman, M. L. 2002, Science, 295, 93

Ahn, K., Shapiro, P. R., Iliev, I. T., Mellema, G., Pen, U.-L. 2008, ApJ, submitted; arXiv:0807.2254

Bennett, O. J., Dickinson, A. S., Leininger, T., \& Gadéa, F. X. 2003, MNRAS, 341, 361

Bate, M. R., Bonnell, I. A., \& Price, N. P. 1995, MNRAS, 277, 362

Bate, M. R. \& Burkert, A. 1997, MNRAS, 288, 1060

Bodenheimer, P. 1995, ARA\&A, 33, 199

Bromm, V., Coppi, P. S., \& Larson, R. B. 1999, ApJ, 527, L5

Bromm, V., Coppi, P. S., \& Larson, R. B. 2002, ApJ, 564, 23

Bromm, V. \& Larson, R. S. 2004, ARA\&A, 42, 79

Bromm, V. \& Loeb, A. 2003, ApJ, 596, 34

Bromm, V. \& Loeb, A. 2004, New Astron., 9, 353

Bruhns, H., et al. 2008, AAS Meeting Abstracts, 212, 03.21

Cen, R. \& Riquelme, M. A. 2007, ApJ, 674, 644

Clark, P. C., Glover, S. C. O., \& Klessen, R. S. 2008, ApJ, 672, 757

Clarke, C. J. \& Bonnell, I. A. 2008, MNRAS, 388, 1171

Cyburt, R. H. 2004, Phys. Rev. D, 70, 023505

Dalgarno, A., Kirby, K., \& Stancil, P. C. 1996, ApJ, 458, 397

Defazio, P., Petrongolo, C., Gamallo, P., \& González, M. 2005, J. Chem. Phys., 122, 214303

Diemand, J., Moore, B., \& Stadel, J. 2005, Nature, 433, 389

Ekström, S., Meynet, G., Chiappini, C., Hirschi, R., \& Maeder, A. 2008, A\&A, accepted; arXiv:0807.0573 
Elmegreen, B. G. 2000, ApJ, 530, 277

Engel, E. A., Doss, N., Harris, G. J., \& Tennyson, J. 2005, MNRAS, 357, 471

Flower, D. R., Le Bourlot, J., Pineau des Forêts, G., \& Roueff, E. 2000, MNRAS, 314, 753

Flower, D. R. \& Harris, G. J. 2007, MNRAS, 377, 705

Galli, D. \& Palla, F. 1998, A\&3A, 335, 403

Gianturco, F. A. \& Gori Giorgi, P. 1996, ApJ, 479, 560

Glover, S. C. O. 2005, Space Sci. Rev., 117, 445

Glover, S. C. O., 2008, in First Stars III, eds. B. O'Shea, A. Heger, \& T. Abel, (New York:AIP), 25

Glover, S. C. O. \& Abel, T. 2008, MNRAS, in press; arXiv:0803.1768

Glover, S., Savin, D., Jappsen, A.-K. 2006, ApJ, 640, 553

Glover, S. C. O. \& Savin, D. W. 2006, Phil. Trans. Roy. Soc. Lond. A, 364, 3107

Glover, S. C. O. \& Savin, D. W. 2008, MNRAS, submitted

Goodwin, S. P., Whitworth, A. P., \& Ward-Thompson, D. 2004, A\&AA, 414, 633

Goodwin, S. P., Whitworth, A. P., \& Ward-Thompson, D. 2004, A\&A, 423, 169

Greif, T. H. \& Bromm, V. 2006, MNRAS, 373, 128

Greif, T. H., Johnson, J. L., Bromm, V., \& Klessen, R. S. 2007, ApJ, 670, 1

Greif, T. H., Johnson, J. L., Klessen, R. S., \& Bromm, V. 2008, MNRAS, 387, 1021

Haiman, Z., Abel, T., \& Rees, M. J. 2000, ApJ, 534, 11

Haiman, Z., Rees, M. J., \& Loeb, A. 1997, ApJ, 476, 458

Haiman, Z., Thoul, A., \& Loeb, A. 1996, ApJ, 464, 523

Heger, A., Woosley, S. E. 2002, ApJ, 567, 532

Jasche, J., Ciardi, B., \& Ensslin, T. A. 2007, MNRAS, 380, 417

Jacobs, T. A., Giedt, R. R., Cohen, N. 1967, J. Chem. Phys., 47, 54

Jappsen, A.-K., Klessen, R. S., Glover, S. C. O., \& Mac Low, M.-M. 2007, ApJ, submitted; arXiv:0709.3530

Johnson, J. L. \& Bromm, V. 2006, MNRAS, 366, 247

Johnson, J. L., Greif, T. H., \& Bromm, V. 2007, ApJ, 665, 85

Johnson, J. L., Greif, T. H., \& Bromm, V. 2008, MNRAS, 388, 26

Kitsionas, S. \& Whitworth, A. P. 2002, MNRAS, 330, 129

Kudritzki, R. P. 2002, ApJ, 2002, 577, 389

Lepp, S. \& Shull, J. M. 1984, ApJ, 280, 465

Machacek, M. E., Bryan, G. L., \& Abel, T. 2001, ApJ, 548, 509

Machida, M. N., Kohji, T., Nakamura, F., \& Fujimoto, M. Y. 2005, ApJ, 622, 39

Mackey, J., Bromm, V., \& Hernquist, L. 2003, ApJ, 586, 1

Mac Low, M.-M., \& Klessen, R. S. 2004, Rev. Mod. Phys., 76, 125

McGreer, I. D. \& Bryan, G. L. 2008, ApJ, accepted; arXiv:0802.3918

Miller, S., Achilleos, N., Ballester, G. E., Geballe, T. R., Joseph, R. D., Prangé, R., Rego, D., Stallard, T., Tennyson, J., Trafton, L. M., \& Waite, J. H., Jr. 2000, Phil. Trans. R. Soc., 358,2485

Mizusawa, H., Omukai, K., \& Nishi, R. 2005, PASJ, 57, 951

Nagakura, T. \& Omukai, K. 2005, MNRAS, 364, 1378

Nakamura, F. \& Umemura, M. 2002, ApJ, 569, 549

Naoz, S., Noter, S., \& Barkana, R. 2006, MNRAS, 373, L98

Neale, L., Miller, S., \& Tennyson, J. 1996, ApJ, 464, 516

Norman, M. L. 2008, in First Stars III, eds. B. O'Shea, A. Heger, \& T. Abel, (New York:AIP), 3

Omukai, K. \& Nishi, R. 1998, ApJ, 508, 141

Omukai, K., Tsuribe, T., Schneider, R., \& Ferrara, A. 2005, ApJ, 626, 627

O'Shea, B. W. \& Norman, M. L. 2007, ApJ, 654, 66

O'Shea, B. W. \& Norman, M. L. 2008, ApJ, 673, 14

Palla, F., Salpeter, E. E., \& Stahler, S. W. 1983, ApJ, 271, 632

Peebles, P. J. E. \& Dicke, R. H. 1968, ApJ, 154, 891

Reed, D. S., Bower, R., Frenk, C. S., Gao, L., Jenkins, A., Theuns, T., \& White, S. D. M. 2005, MNRAS, 363, 393 
Ricotti, M., Gnedin, N., \& Shull, J. M. 2002, ApJ, 575, 49

Ripamonti, E., Haardt, F., Ferrara, A., \& Colpi, M. 2002, MNRAS, 334, 401

Ripamonti, E. \& Abel, T. 2004, MNRAS, 348, 1019

Ripamonti, E. 2007, MNRAS, 376, 709

Salvaterra, R., Ferrara, A., \& Schneider, R. 2004, New Astron., 10, 113

Saslaw, W. C. \& Zipoy, D. 1967, Nature, 216, 976

Savin, D. W., Krstic, P. S., Haiman, Z., \& Stancil, P. C. 2004, ApJ, 606, L167; erratum ApJ, 607, L147

Shapiro, P. R. \& Kang, H. 1987, ApJ, 318, 32

Shchekinov, Y. A. \& Vasiliev, E. O. 2006, MNRAS, 368, 454

Stacy, A. \& Bromm, V. 2007, MNRAS, 382, 229

Suchkov, A. A. \& Shchekinov, Y. A. 1977, Sov. Astr. Lett., 3, 297

Suchkov, A. A. \& Shchekinov, Y. A. 1978, Sov. Astr. Lett., 4, 164

Tan, J. C. \& McKee, C. F. 2008, in First Stars III, eds. B. O'Shea, A. Heger, \& T. Abel, (New York:AIP), 47

Tegmark, M., Silk, J., Rees, M., Blanchard, A., Abel, T., \& Palla, F. 1997, ApJ, 474, 1

Truelove, J. K., Klein, R. I., McKee, C. F., Holliman, J. H., Howell, L. H., \& Greenough, J. A. 1997, ApJ, 489, L179

Turk, M. J., Abel, T., \& O'Shea, B. W. 2008, in First Stars III, eds. B. O'Shea, A. Heger, \& T. Abel, (New York:AIP), 16

Umeda, H. \& Nomoto, K. 2002, ApJ, 565, 385

Wise, J. H. \& Abel, T. 2007, ApJ, 671, 1559

Yoshida, N., Abel, T., Hernquist, L., \& Sugiyama, N. 2003, ApJ, 592, 645

Yoshida, N., Omukai, K., Hernquist, L., \& Abel, T. 2006, ApJ, 652, 6

Yoshida, N., Omukai, K., \& Hernquist, L. 2007, ApJ, 667, L117

Yoshida, N., Oh, S. P., Kitayama, T., \& Hernquist, L. 2007, ApJ, 663, 687

Zemke, W. T. \& Stwalley, W. C. 1980, J. Chem. Phys., 73, 5584 